\title{
Confined Blood Chimerism in Monochorionic Dizygotic Twins Conceived Spontaneously
}

\author{
Takashi Kanda, MD ${ }^{1}$ Masanobu Ogawa, MD, $\mathrm{PhD}^{2} \quad$ Kazuo Sato, MD, $\mathrm{PhD}^{1}$ \\ ${ }^{1}$ Department of Pediatrics, Clinical Research Institute, National \\ Hospital Organization Kyushu Medical Center, Fukuoka, Japan \\ 2 Department of Obstetrics and Gynecology, Clinical Research \\ Institute, National Hospital Organization Kyushu Medical Center, \\ Fukuoka, Japan

\begin{abstract}
Address for correspondence Takashi Kanda, MD, Department of Pediatrics, Clinical Research Institute, National Hospital Organization Kyushu Medical Center, 1-8-1 Jigyohama, Chuo-ku, Fukuoka 810-8563, Japan (e-mail: ktakashi@qmed.hosp.go.jp).
\end{abstract}

Am J Perinatol Rep 2013;3:33-36.

\author{
Abstract \\ Keywords \\ - monochorionic \\ dizygotic twins \\ - spontaneous \\ pregnancy \\ - confined blood \\ chimerism \\ - freemartinism
}

Traditionally, monochorionicity has been regarded as synonymous with monozygosity. However, several recent cases of monochorionic dizygotic twins have shown that monochorionic twins can be dizygous. We report a rare case of monochorionic diamnionic, gender-discordant twins who were conceived spontaneously. Initially, a monochorionic placenta was diagnosed by ultrasonography at 8 weeks of gestation and then confirmed by pathology after delivery. The twins had different genders. A comparison of cytogenetic analyses using peripheral blood lymphocytes and skin fibroblasts revealed that chimerism was confined to blood cells. We have experienced two cases of monochorionic dizygotic twins since 2003. These cases suggest that monochorionic dizygotic twins are not as rare as previously thought.
Traditionally, monochorionic twins have been considered to be monozygous. This concept is founded upon two reports that evaluated placental pathology and serological markers of zygosity. ${ }^{1,2}$ Although a case of monochorionic placentation with heterosexual twins was reported by Nylander and Osunkoya, ${ }^{3}$ this report lacked cytogenetic confirmation of zygosity. Therefore, the possibility of a monochorionic placenta supporting dizygotic twins is not extensively recognized. Since Souter et al reported dizygous monochorionic twins in whom both monochorionicity and dizygosity were confirmed by placental pathology and cytogenetic analysis of skin fibroblasts in 2003, ${ }^{4}$ an increasing number of such cases has been reported in recent years. In most such cases. the pregnancies were achieved by assisted reproductive technology (ART). Therefore, it has been thought that these exceptional cases are associated with ART. We report a rare case of monochorionic dizygotic twins in a spontaneous pregnancy complicated by confined blood chimerism.

\section{Case Report}

A 28-year-old healthy primigravida woman conceived naturally. An ultrasound evaluation performed at 8 weeks of

received

May 3, 2012

accepted after revision

September 23, 2012

published online

January 2, 2013

gestation indicated monochorionic and diamnionic twins. At 24 weeks of gestation, an ultrasound evaluation showed that the fetuses were discordant for gender despite having a monochorionic placenta. There was a discordant growth pattern, but no other signs of twin-twin transfusion syndrome (TTTS) were present.

The infants were delivered vaginally at 36 weeks and 1 day of gestation. Twin 1 was a phenotypically normal healthy boy with a birth weight of 2944 g. Twin 2 was a phenotypically normal healthy girl with birth weight of 2502 g. Sexual ambiguity was not present in either infant. An ultrasound evaluation of the girl performed at 4 months of age revealed the presence of bilateral ovaries and a uterus. Pathology of the placenta showed that the septum consisted of two layers of amnion but lacked layers of chorion (-Fig. 1). It was confirmed that the placenta was monochorionic and diamnionic. Cytogenetic analysis of blood lymphocytes obtained when the twins were 1 month of age showed 46,XX[5]/46,XY[25] in the boy and 46,XY[17]/46,XX[13] in the girl. At 6 months of age, analysis of skin fibroblasts showed normal male and female karyotypes. Florescent in situ hybridization analysis of blood lymphocytes at 1 year showed that the chimerism was

Copyright $\odot 2013$ by Thieme Medical Publishers, Inc., 333 Seventh Avenue, New York, NY 10001, USA. Tel: +1(212) 584-4662.
DOI http://dx.doi.org/ $10.1055 / \mathrm{s}-0032-1331377$. ISSN 2157-6998. 


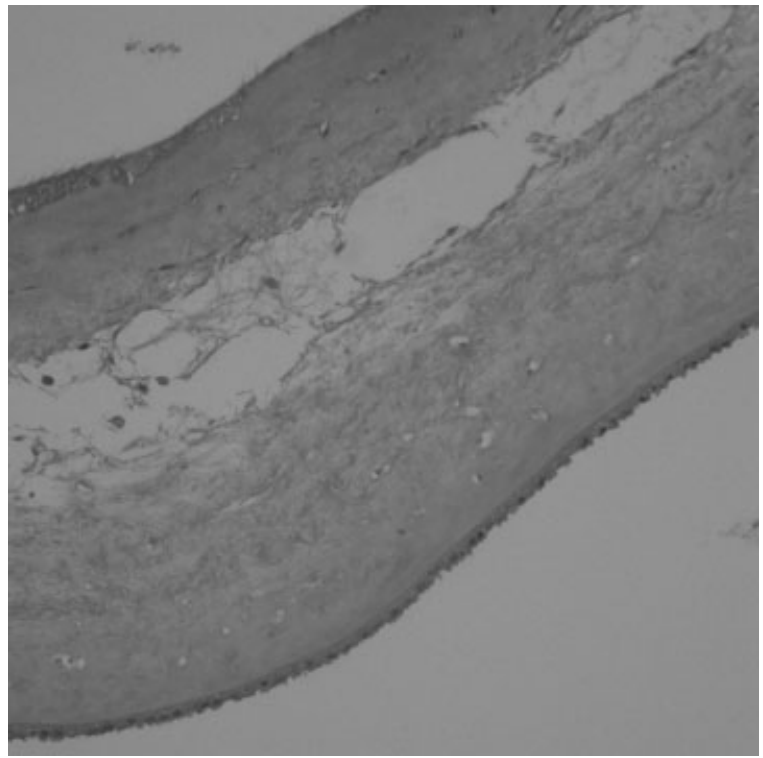

Fig. 1 The dividing membrane is shown. The septum consists of two layers of amnion, with the absence of chorion. Hematoxylin and eosin stain, $\times 400$.

persistent. Blood group typing undertaken with antisera for blood group antigens and flow cytometry analysis of the erythrocytes showed that blood groups were the same in both infants. Written informed consent for cytogenetic analysis and this case report were obtained from the parents.

\section{Discussion}

Monochorionic dizygotic twins in a spontaneous pregnancy are extremely rare. Three cases of monochorionic dizygotic twins in spontaneous pregnancies have been published. 3,5,6 Two of these cases lacked pathological confirmation of monochorionicity or cytogenetic confirmation of dizygosity. We report the second case in which monochorionicity and dizygosity were confirmed by pathology and cytogenetic analysis. Hypotheses for monochorionic dizygotic placentation have previously been proposed. Miura and Niikawa suggested that ART techniques, such as assisted hatching of the zona pellucida, implantation of embryos close together by simultaneous embryo transfer, use of fertilized eggs that have developed to the blastocyst stage, and cell culture procedures that lead to changes in the nature of the cell surface might all increase the chance of embryo fusion. ${ }^{7}$ This "chance hypothesis" suggests that this phenomenon is extremely rare in spontaneous pregnancies. The other hypothesis is that the twins arise via binovular follicle fertilization. Binovular follicles, in which two oocytes are present within a single zona pellucida, were reported in adult women in both nonstimulated and stimulated cycles. ${ }^{8}$ One or both of the oocytes in a binovular follicle were reported to be fertilized in vivo. ${ }^{9}$ Because therapy with exogenous gonadotropic hormones increases the frequency of binovular follicles, this phenomenon is rare in nonstimulated cycles. If intracytoplasmic sperm injection (ICSI) is performed on both oocytes from a binovular follicle, dispermic fertilization of a binovular follicle can occur. Therefore, cases with a spontaneous pregnancy and cases arising from ICSI make this hypothesis unlikely. ${ }^{10}$ An alternative hypothesis has been proposed. Nylander and Osunkoya suggested that the chorions might fuse early in pregnancy and then complete disintegration of the two chorionic layers in the dividing septum would occur later. ${ }^{3}$ Tarkowski and Wojewodzka reported spontaneous in vitro fusion of mammalian embryos under certain conditions during the preimplantation period. $^{11}$

Blood chimerism occurs by the exchange of blood stem cells between fetuses through vascular anastomoses of the placenta in early pregnancy. It has been suggested that this exists persistently because twins are immunologically tolerant to each other and it has been suggested that this phenomenon is more common than expected. ${ }^{12}$ Although this phenomenon was also reported among dichorionic twins, ${ }^{13}$ such cases are rare because anastomotic communication is rarely found within dichorionic placentas. ${ }^{14}$ We have experienced two cases of monochorionic dizygotic twins, including the current case, among 89 monochorionic pregnancies since 2003. The other case was monochorionic dizygotic twins conceived following ICSI and complicated by TTTS in 2003. A 27-year-old primigravida woman who conceived following ICSI delivered a phenotypically normal boy with a birth weight of $1414 \mathrm{~g}$ and a phenotypically normal girl with a birth weight of $942 \mathrm{~g}$ at 30 weeks and 6 days of gestation by cesarean section. Sexual ambiguity was not present in either infant. Both monochorionicity and dizygosity were confirmed by pathology and cytogenetic analysis. Therefore, we speculate that monochorionic dizygotic pregnancies are not as rare as previously thought.

A freemartin is a sterile and masculinized female mammal born as a twin to a male, and it shows masculinization of the female reproductive tract to varying degrees. A freemartin arises when vascular connections form between the placentas of developing heterosexual twin fetuses. In heterosexual twins, male and female cells pass from one sibling to the other, resulting in XX/XY chimeras. The masculinized phenotype is not due to the male cells themselves, but is primarily an effect of circulating müllerian inhibiting substance (MIS, also termed anti-Müllerian hormone). Newborn males and freemartins have high levels of MIS in plasma, and females have much lower levels. The freemartin represents the most frequent form of intersexuality found in cattle and is less common in other species. ${ }^{15}$ In our case, the twin girl did not display freemartinism by an ultrasound evaluation. It has been suggested that freemartinism does not occur in humans because the pathogenetic effects of vascular anastomoses differ between humans and cattle among sex discordant, monochorionic twins. Bogdanova et al reported that a trisomy 21 female with aplasia of the uterus and the Fallopian tubes was a freemartin. ${ }^{16}$ This case lacked evaluation of serum MIS levels.

In conclusion, the current case results suggest that monochorionic dizygotic twins can occur in a spontaneous pregnancy. It appears that this phenomenon can occur more frequently following ART. Our two cases among 89 
monochorionic pregnancies suggest that this phenomenon is more common than originally thought; however, the exact incidence is unclear. Therefore, further studies to estimate a reliable incidence and etiology of monochorionic dizygotic twins are recommended to redefine the theories relating to placentation and zygosity.

\section{References}

1 Vlietinck R, Derom C, Derom R, Van den Berghe H, Thiery M. The validity of Weinberg's rule in the East Flanders Prospective Twin Survey (EFPTS). Acta Genet Med Gemellol (Roma) 1988;37: 137-141

2 Husby H, Holm NV, Gernow A, Thomsen SG, Kock K, Gürtler H. Zygosity, placental membranes and Weinberg's rule in a Danish consecutive twin series. Acta Genet Med Gemellol (Roma) 1991;40:147-152

3 Nylander PP, Osunkoya BO. Unusual monochorionic placentation with heterosexual twins. Obstet Gynecol 1970;36:621-625

4 Souter VL, Kapur RP, Nyholt DR, et al. A report of dizygous monochorionic twins. N Engl J Med 2003;349:154-158

5 Shalev SA, Shalev E, Pras E, et al. Evidence for blood chimerism in dizygotic spontaneous twin pregnancy discordant for Down syndrome. Prenat Diagn 2006;26:782-784

6 Hackmon R, Jormark S, Cheng V, O'Reilly Green C, Divon MY. Monochorionic dizygotic twins in a spontaneous pregnancy: a rare case report. J Matern Fetal Neonatal Med 2009;22: 708-710
7 Miura K, Niikawa N. Do monochorionic dizygotic twins increase after pregnancy by assisted reproductive technology? J Hum Genet 2005;50:1-6

8 Ron-El R, Nachum H, Golan A, Herman A, Yigal S, Caspi E. Binovular human ovarian follicles associated with in vitro fertilization: incidence and outcome. Fertil Steril 1990;54:869-872

9 Vicdan K, Işik AZ, Dağli HG, Kaba A, Kişniş̧̧i H. Fertilization and development of a blastocyst-stage embryo after selective intracytoplasmic sperm injection of a mature oocyte from a binovular zona pellucida: a case report. J Assist Reprod Genet 1999;16: 355-357

10 Ekelund CK, Skibsted L, Søgaard K, et al. Dizygotic monochorionic twin pregnancy conceived following intracytoplasmic sperm injection treatment and complicated by twin-twin transfusion syndrome and blood chimerism. Ultrasound Obstet Gynecol 2008;32:832-834

11 Tarkowski AK, Wojewodzka M. A method for obtaining chimaeric mouse blastocysts with two separate inner cell masses: a preliminary report. J Embryol Exp Morphol 1982;71:215-221

12 van Dijk BA, Boomsma DI, de Man AJ. Blood group chimerism in human multiple births is not rare. Am J Med Genet 1996;61: 264-268

13 Jang JH, Jung H, Kim JH, Park WS, Kim SH. Blood chimerism in a dizygotic dichorionic pregnancy. Korean J Lab Med 2010;30:521-524

14 Robertson EG, Neer KJ. Placental injection studies in twin gestation. Am J Obstet Gynecol 1983;147:170-174

15 Padula AM. The freemartin syndrome: an update. Anim Reprod Sci 2005;87:93-109

16 Bogdanova N, Siebers U, Kelsch R, et al. Blood chimerism in a girl with Down syndrome and possible freemartin effect leading to aplasia of the Müllerian derivatives. Hum Reprod 2010;25:1339-1343 